\title{
Reconstruction Technology Application of Reverse Engineering in Centrifugal Pump Inducer
}

\author{
Li Zhu ${ }^{\mathrm{a}}$, Xi Zhao ${ }^{\mathrm{b}}$, Guoqing Ma ${ }^{\mathrm{c}}$ \\ School of Energy and Environment, Xihua University, Chengdu 610039, China \\ azohouoloi@163.com, bgabuer@yeah.net, 'librama@yeah.net
}

Keywords: reverse engineering, centrifugal pump inducer, secondary fairing, section feature.

\begin{abstract}
For the 3D model reconstruction of centrifugal pump inducer based on reverse engineering, the hub and the blade of centrifugal pump inducer are reconstructed respectively according to their structures. This paper firstly introduces the laser scanning method by which the rotary-future based model reconstruction of hub is achieved with the technology of the cross- sectional constraints. And then taking advantage of the raw point cloud data of inducer blade, the secondary point cloud fairing is used on the blade in the close-to-streamline direction and axis direction. After the secondary point cloud fairing, the B-spline curve fitting can be realized in the two different directions, thus getting the corresponding blade through layout with the use of surface meshes. Finally, combining the reconstruction of hub with that of blade, we can get the whole 3D model of centrifugal pump inducer. This paper also explains the accuracy detection for reconstruction model to illustrate the accuracy and feasibility of this method.
\end{abstract}

\section{Introduction}

The properties of centrifugal pump inducer play an important role in the cavitation resistance of centrifugal pump and solving the problem of cavitation. Inducer has widely applied in high-speed pump and centrifugal pump which requires a good performance in cavitation resistance [1]. The traditional design of inducer is no longer able to satisfy the market demanding various personalized products. An obvious case is the drawing of both floor plan and airfoil expansion graph of inducer, whose measurement takes a long cycle, leading to relatively more errors. Reverse Engineering, as a method of reconstructing three-dimensional CAD model based on measurement data, provides a rapid and reliable way for the development and innovation of such related products [2].

\section{Method of centrifugal pump inducer reconstruction}

The reverse engineering of centrifugal pump inducer mainly consists of point cloud data acquisition and preprocessing, surface reconstruction and accuracy detection of three-dimensional model reconstruction. The surface reconstruction is the key step in the whole reverse engineering, which plays a decisive role in the accuracy and precision of three-dimensional model reconstruction. In recent years, the domestic and foreign scholars have conducted a thorough research for the surface reconstruction. The two literatures [3, 4] respectively take the biquadrate Bezier method, minimum energy method as well as NURBS method to study the surface reconstruction, providing reference for the practical engineering of surface reconstruction. There are two kinds of surface construction: one is triangular facet-based free-form surface reconstruction, the other is feature-based parameter reconstruction. In essence, the former is a "point-triangular-surface patches" operation process that is a fast surface reconstruction method and mostly applied in those model reconstructions whose contour features are easy to be divided. However, the precision of the free-form surface reconstruction is not high. On the contrary, the precision of feature-based parameter reconstruction is relatively higher, for it's a "point-line-surface" operation process which can really reflect the designer's idea.

Concerning the engineering practice, the ideas for the centrifugal pump inducer reconstruction introduced in this paper is a combination of the above two kinds of surface reconstruction method and 
a division of hub reconstruction and blade reconstruction respectively according to the structural characteristics of the inducer. On one hand, because of the apparent rotary characteristics of hub, slicing method is used to obtain the cross-section point cloud data to fit the rotary characteristics and thus the three-dimensional model of hub is achieved by the tensile way. On the other hand, since the spatial characteristics of the blade are irregular and distorting and in order to ensure the overall fairness, secondary point cloud fairing is used on the blade in the close-to-streamline direction and axis direction. After the point cloud fairing, the B-spline curve fitting can be realized in the two different directions, thus getting the corresponding blade through layout with the use of surface meshes. Finally, combining the reconstruction of hub with that of blade, we can get the whole 3D model of centrifugal pump inducer.

\section{Point cloud data acquisition and centrifugal pump inducer preprocessing}

Point cloud data acquisition of centrifugal pump inducer. The method of data acquisition used in reverse engineering can be classified into two kinds: contact measurement and non-contact measurement. In this paper, point cloud data acquisition is achieved by the use of the handy laser scanner. The system of handy laser scanner cannot recognize the surface condition of the marked point, the covering area of which will be automatically filled in a planar form. In order to improve the scanning precision of point cloud data, it's suggested that the distance between two marked points should not be less than $20 \mathrm{~mm}$, the distance between the marked point and the edge not less than $10 \mathrm{~mm}$, and that the marked points should not be posted in the featured parts or the area where the curvature changes more rapidly and frequently. The scanning process is conducted on the principle called "first overall, then parts" [5]. It means that after getting most of the point cloud data, more time should be spent to scan the detailing parts from different angles. Taking the surface-based optimal algorithm technology used in software measurement tool VXscan into consideration, a longer time of scanning needs to be stayed in the corner position in order to get more accurate and complete point cloud data. The measurement condition on the spot is shown in figure1.
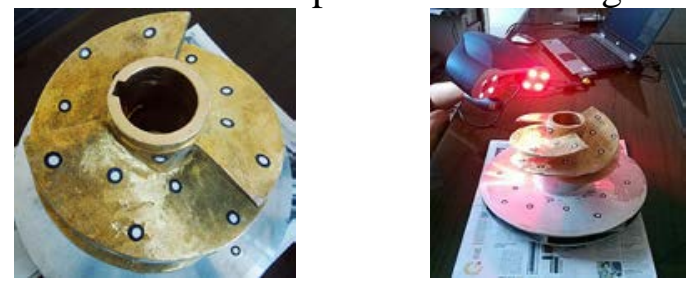

Fig.1 Field measurement for centrifugal pump inducer

Point cloud data preprocessing of centrifugal pump inducer. Laser triangulation, the data acquisition method used in this paper, can obtain a huge amount of inducer data through laser scanning, including massive abnormal points which deviated from the measurement data. Moreover, the sharp edge of parts has partially become blind spots and gaps for the laser scanning. All the problems mentioned above have together turned out to be one of the most seriously influencing factors on the precision and fairness of surface reconstruction. In order to solve these problems and improve the quality of the reconstructed surface, point cloud data preprocessing before surface reconstruction must be conducted. Figure 2 is a flow diagram of inducer point cloud data preprocessing adopted in this paper. Figure 3 is a comparison diagram: before the preprocessing is shown as Figure 3 (a), after the preprocessing is shown as Figure 3 (B).

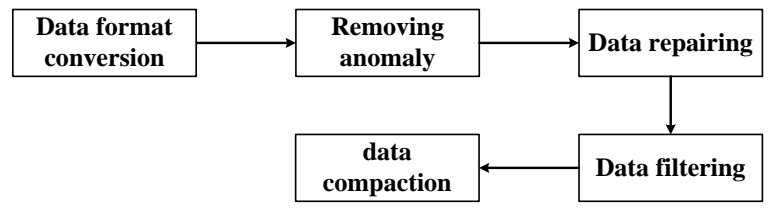

Fig.2 Pretreatment processes

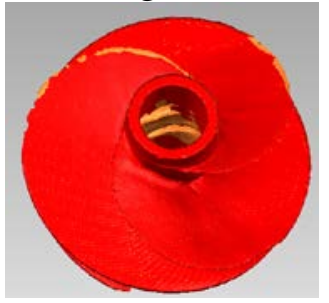

a

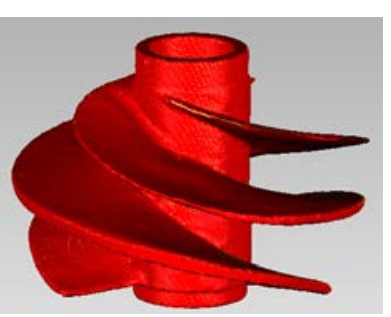

b

Fig. 3 Pretreatment process for point clouds 


\section{The Reconstitution of Centrifugal Pump Inducer Model}

The Reconstitution of Centrifugal Pump Inducer Hub. As we know, cross sectional point cloud is the foundation of reconstitute inducer hub when it rotating and stretching applets. The acquisition of the cross section point cloud data steps are as follows:

Step1. Determination of the axis direction of the hub. The intercepted data of hub end face matching to the plane, then the plane as the datum to the parallel surface intercept shaft hole data and fitting for the round. According to the center of the circle fitting for a straight line to get the axial direction, as shown in figure 4.

Step2. Interception of hub point cloud data based on slice method of axial direction. To improve the accuracy of reconstructed model needs to reference multiple axial surfaces, as shown in figure 5 .

Step3. Rotation of the axial surface to the same hub point cloud data surface and filter its data. According to the curvature variation, divide the point cloud data into four blocks and then using the Gaussian filter to filter the data.

Through above methods to get the rotary section features and center axis, then around the axis rotate for 360 degree to get the hub three-dimensional model, as shown in figure 6 .

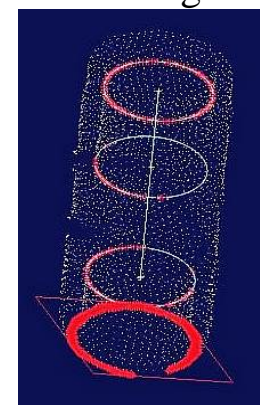

Fig.4 Axis fitting of the hub

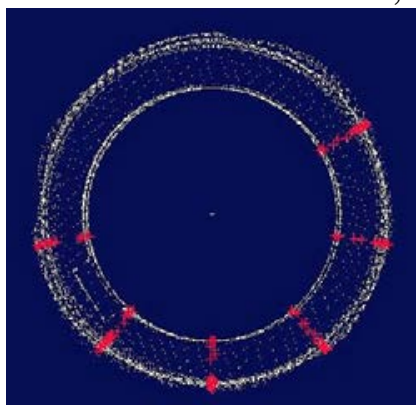

Fig.5 Point cloud slices with model of inducer hub

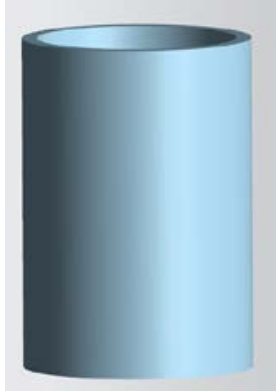

Fig. 6 Three-dimensional axis

Centrifugal Pump Inducer Blade Reconstruction. The reconstruction of inducer blade can be obtained through the section groups which axis-centered and get the equal angle of both sides of the borderline. Then on this basis to obtain the complete 3D model of blade. But this method cannot accurately meet the fairing requirements of the flow direction and hub direction. The section contour data is shown in figure 7.

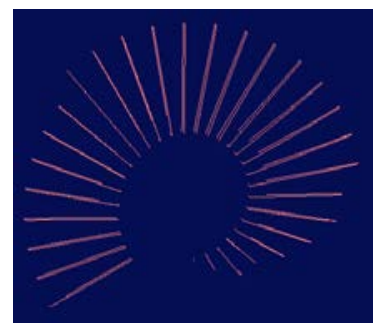

Fig.7 Profile data of inducer section

The surface meshes $\mathrm{u}, \mathrm{v}$ are respectively taken the direction of near-surface and axial direction. As shown in figure 8, extract the single inducer blade point cloud data and based on the reconstruction hub cylinder surface to make the concentric cylindrical surface. Then cutting the blade point cloud. Point clouds are faired by B-spline curve fitting in the Gauss filter to filter the streamline point cloud and least square method [6]. The $\mathrm{u}, \mathrm{v}$ direction curves is obtained through the establishment of point cloud data surface group which centered the hub axis, and extract the intersection of $\mathrm{u}, \mathrm{v}$ direction and B spline curves. Then adopting the least squares method again fitting the B spline curve, as shown in figure 9. Along the axis direction project the original point cloud data to the same plane, extract the blade margin part of the point cloud fitting for B spline curve. Then stretch the curve along the axis direction to get the blade margin surface. The final inducer model can be obtained by extending and cutting when operates on the all sides of blade, as shown in figure 10. 


\section{Centrifugal Pump Inducer Reconstruction Model Accuracy Detection}

The surface quality evaluation of fluid machinery parts such as over-current components, should consider not only the precision of surface reconstruction but also the surface fairness. The detection of surface precision is comparing the reconstruction of blade surface CAD model with the original point cloud data. In this paper, the smoothing analysis apples the isophote method [7], as can be seen in figure 11, the smooth meet the requirements of isophote uniform, smooth, but not the twisting, bending phenomenon. As can be seen from figure 12, the maximum deviation of the reconstruction centrifugal pump blade surface is $0.876 \mathrm{~mm}$; the average deviation is $0.060 \mathrm{~mm}$. The existence of larger error is caused by the compensation errors which generate from the measurement markers. The error of blade margin, such as leading-edge and trail-edge, is caused by the process of laser scanner system with smooth surface recognition affect the reconstructed precision. The standard deviation of CAD model is $0.175 \mathrm{~mm}$ which meets the practical engineering requirements.

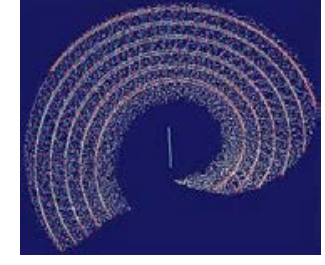

Fig.8 Point cloud curve fitting on flow direction

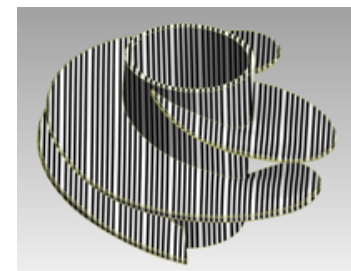

Fig.11Text for fairing

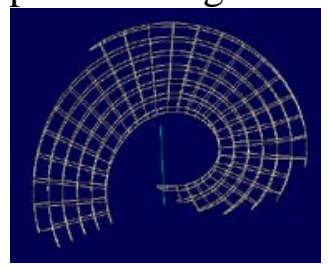

Fig.9 Surface groups on $\mathrm{u}$ and $\mathrm{v}$ directions

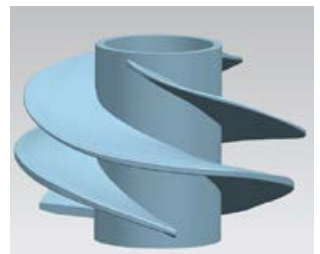

Fig.10 Three-dimensional model of inducer

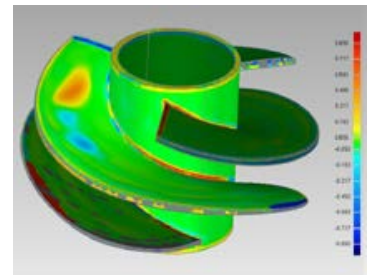

Fig. 12 Test for reconstructed surface accuracy

\section{Conclusions}

Based on the traditional design method of "hydraulic, geometric, structure, test" is unable to meet the practical engineering needs. The inducer three-dimensional model is reconstructed with the technology of the cross - sectional constraints and induction rotor blade is used the secondary smoothing on the flow direction in the near-surface and axial direction through laser scanning method. For the reconstruction of the hub, adopt section curve of extract feature simplifies the difficulty and improve the precision and efficiency greatly; For the blade, the surface meshes by constructing $\mathrm{u}, \mathrm{v}$ direction curve group to loft to get blade which constructed to meet the smoothness of flow direction and axial direction. Through the detection of reconstructed model precision demonstrates that this method provides a fast and accurate way for centrifugal pump inducer reconstruction.

\section{References}

[1]Xu Chengbo, Luo Dazhang. Pump Technology, 1998, (04).

[2]Wang Xiao, Liu Huixia, Ling Jiahong. Chemical industry publishers. 2004.

[3]Wang Chunlin, Yuan Chunyuan, et al. Modeling research for fluid mechanical blade shape based on Bezier function [J]. Pump technology, 2005, 1:10-11.

[4]Qi Difei, Yang Jinsong, Liu Guogan, et al. Three-dimensional reconstruction for plane blades with NURBS [J]. Optics and Precision Engineering, 2001, 9 (3) :223-225.

[5]Wu Lushen, Gao Hongwei, Meng Fanwen. NURBS Surface reconstruction and point clouds surface error analysis [J]. Laser and Infrared, 2010, 4 (10):1131 -1135.

[6]Zhou Minghua, Wang Guozhao. Based on genetic algorithm B-spline curves and B-spline Bézeir curves least squares fitting [J]. Computer Research and Development, 2005, (42) :134-143.

[7]Ji Xiaogang, Gong Gongrong. Computer Application Research, 2007, 24 (3): 85-88. 